\title{
Use of Vibrational Optical Coherence Tomography to Characterize Skin Lesions and a Congenital Nevus
}

\author{
Frederick H Silver ${ }^{1,2 *}$, Ruchit G Shah ${ }^{2}$ and Nikita Kelkar ${ }^{2}$ \\ ${ }^{1}$ Department of Pathology and Laboratory Medicine, Robert Wood Johnson Medical School, Rutgers, The State University \\ of New Jersey, Piscataway, NJ, USA \\ ${ }^{2}$ OptoVibronex, LLC., Mt. Bethel, Pa, USA
}

"Corresponding author: Frederick H. Silver, Department of Pathology and Laboratory Medicine, Robert Wood Johnson Medical School, Rutgers, The State University of New Jersey, Piscataway, NJ-08854, Tel: +6104282173; E-mail: silverfr@rutgers.edu; fhsilver@hotmail.com

\begin{abstract}
We have used vibrational optical coherence tomography (VOCT) to image and measure the mechanical properties of normal skin and skin lesions. It is observed that in a congenital nevus and normal skin, the cellular epidermis is qualitatively not as bright as in skin lesions including basal cell carcinoma, actinic keratosis and a melanocytic nevus. Melanin and cytochrome c oxidase are reported to attenuate the reflection of near-infrared light at a wavelength of $810 \mathrm{~nm}$ and therefore may explain the reduced reflection of light in a congenital nevus and normal skin under conditions where cytochrome c oxidase levels would be expected to be high.

Our results suggest that the melanin and cytochrome c oxidase levels found in congenital nevus and skin lesions may influence the observed pixel density observed in OCT images. For this reason, a correction for the content of these components in the skin must be considered before quantitative pixel measurements can be correctly interpreted. Additional measurements of pixel density along with the moduli of cellular and collagenous components in skin and skin lesions are needed to further interpret the significance of "virtual biopsies" made using VOCT.

Keywords: Optical coherence tomography; Vibrational optical coherence tomography; Epidermis; Dermoscopy; Ultrasonography; Refractive confocal microscopy; Dermis; Pixel density; Modulus; Resonant frequency
\end{abstract}

Received Date: May 14, 2019; Accepted Date: June 25, 2019; Published Date: July 02, 2019

\section{Introduction}

A number of methods have been used to noninvasively image skin and skin lesions including dermoscopy, reflective confocal microscopy, ultra-sonography and optical coherence tomography [1-7]. They can be used not only for diagnosis but also to

Citation: Frederick H Silver, Use of Vibrational Optical Coherence Tomography to Characterize Skin Lesions and a Congenital Nevus. J Clin Cases Rep 2(4): 109-118. DOI: https://doi.org/10.46619/joccr.2019.2-1051

(C) 2019 Tridha Scholars 
http://www.tridhascholars.org | October-2019

choose the best therapy to follow a patient's response to treatment in vivo [1]. These methods are complementary to each other producing an image of skin and skin lesions; however, they would be extended by the addition of other physical data that reflect the overall properties of the extracellular matrix [8-19].

Dermoscopy is a noninvasive microscopic technique based on the use of incident light to collect an image minimizing specular reflectance [1]. A combination of dermoscopy and whole-body photography enables mapping of all lesions and their evolution over time. This technique is useful for both pigmented and non-pigmented lesions and yields a magnification of about $20 \mathrm{x}$ $[1,4,7]$. However, dermoscopy alone cannot give information concerning the cellular details such as the extent of mitoses as well as images below the surface of the skin.

Confocal microscopy makes possible a real-time examination of skin and skin lesions. The technique requires a skilled technician and provides a resolution of between 0.5 and $1.0 \mu \mathrm{m}$ [1]. Using this technique, a series of optical sections from the epidermis to the papillary dermis is obtained. These images have been used to refine melanoma diagnosis to be more accurate and provide qualitative information concerning cells, blood vessels and collagen. The images are far superior to those obtained with ultrasound.

Ultrasonography (USG) is based on the reflection of sound at boundaries between tissues of different densities. It uses sound at frequencies between $15 \mathrm{MHz}$ and $20 \mathrm{MHz}$ and the main clinical use is the preoperative assessment of the depth of invasion of melanomas and basal cell carcinomas [3]. The images obtained using high-frequency ultrasound are not a sharp as are those obtained with other techniques, but ultrasound offers the ability to obtain some quantitative information about the tissues being observed.

Optical coherence tomography (OCT) is a technique that creates an image based on interference between incident and reflected light beams generating serial axial images as deep as the reticular dermis [2]. It creates an image with a resolution of between 10 and $20 \mu \mathrm{m}$. Based on OCT images it has been reported that malignant melanomas rarely exhibit a clear epidermal-dermal junction zone when compared with benign nevi [18].

Recently, we have introduced the use of vibrational OCT (VOCT) to characterize a number of tissues in vitro and in vivo [817]. VOCT combines the imaging power of OCT with the ability to quantitatively measure tissue stiffness. Tissue stiffness has been reported to be correlated with changes in cancer cells stiffness; increased tissue stiffness (also termed modulus) has been shown to be a characteristic of potential tumor metastasis [19].

All of these methods give images of skin and skin lesions; however, only VOCT provides calibrated physical data to evaluate the changes in properties of extracellular matrix (ECM) that occur during diseases and wound healing [8-17]. Under normal conditions, the epidermis is thin and the mechanical properties cannot be measured. However, when a benign or malignant skin lesion forms, it is possible to measure the mechanical properties of the epidermis since the epidermis thickens and cellcell interactions affect tissue properties [17,20].

In this paper, we discuss the use of VOCT to image and measure changes in the mechanical properties of skin and skin lesions. Our results suggest that VOCT in concert with dermoscopy and refractive confocal microscopy can be used to improve our 
understanding of skin pathology that occurs in disease based on the measurement of tissue modulus and image pixel intensities.

\section{Methods}

\section{In Vitro Calibration Curve Construction Using Measurements on ECMs}

Vibrational optical coherence tomography (VOCT) is a new technique developed in the Department of Pathology at Robert Wood Johnson Medical School, Rutgers, the State University of New Jersey. It uses near infrared light and audible sound to image and measure the resonant frequency of tissues $[8-17,20]$. The resonant frequency of a tissue is directly related to the tissue stiffness as described previously [8-17, 20].

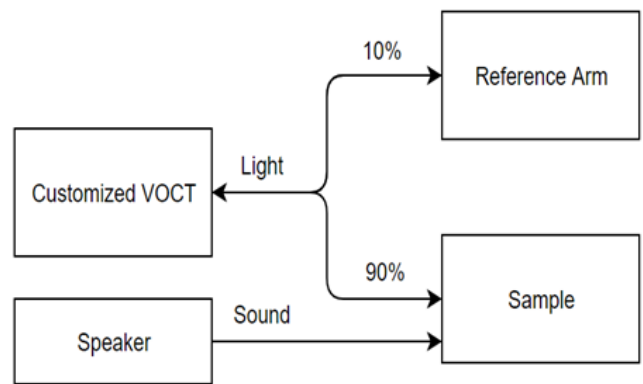

Figure 1: Diagram illustrating the setup used to collect images and measure the mechanical properties of tissues using vibrational optic coherence tomography (VOCT). In OCT, an infrared light beam is split into two components. One beam is directed to a detector and mixed with reflected light from the sample. An image is collected in the scanning mode. In the B mode, after a sinusoidal sound wave is applied, the light is fixed on a single point and the frequency at which the maximum displacement occurs is measured. The modulus is calculated using a calibrations curve (Figure 3).
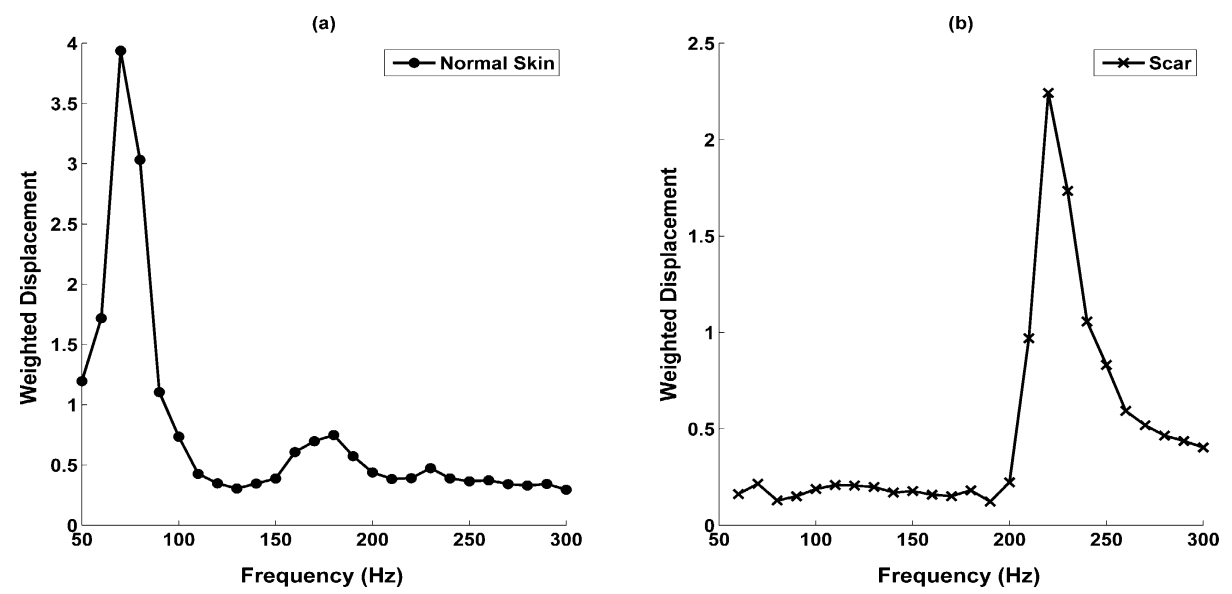

Figure 2: Weighted displacement versus frequency curve for normal skin abd scar tissue used to determine the tissue modulus. The modulus is determined from the frequency at which the maximum displacement occurs using the tissue thickness and the calibration curve shown in Figure 3. The resonant frequency of normal skin is lower than that of scar tissue resulting in a higher modulus for scar using Figure 3 (Table 1).

A schematic diagram of VOCT is shown in (Figure 1). An image of the sample is first obtained using near infrared light with the OCT operating in the scanning mode. A frequency generator is then turned on that is connected to a speaker, and audible sound impinges on the tissue providing a transverse deflection of the surface. The transverse deflection of the tissue is 
http://www.tridhascholars.org | October-2019

measured as a function of the applied sound frequency in the B mode at a fixed point. The resonant frequency is defined as the frequency at which the maximum sample deflection is observed as is illustrated in (Figure 2).

A calibration curve of modulus values for control ECMs was constructed by comparing moduli measured using conventional tensile testing and VOCT measurements in vitro as previously described [8-17]. The constructed calibration curve shows a one-to-one relationship between tensile and vibrational moduli measured on the same samples including decellularized human dermis, pig skin, and bovine cartilage [8-17]. The resonant frequency was determined after correction for the resonant frequencies exhibited by the speaker, sample holders and any interference due to line fluctuations [8-17].

\section{VOCT Measurements}

\section{Image Collection}

OCT cross-sectional images were obtained using a modified OQ Labscope (Lumedica Inc., Durham, NC) [11-17]. The images were collected in the scanning mode. The original grayscale OCT images of skin and skin lesions were pseudo-color-coded based on the pixel intensities to provide better images of the tissue components. The enhanced OCT images used darker colored (blue and purple) regions to reflect lower pixel intensities while the lighter (yellowish) regions reflected higher pixel intensity regions.

\section{OCT and Vibrational Analysis in vivo}

Transverse sample displacement was generated by placing a speaker next to the skin as discussed previously [8-17]. The spectral-domain optical coherence tomography (SD-OCT) system uses a fiber-coupled super luminescent diode light source with an $810 \mathrm{~nm}$ center wavelength and $100 \mathrm{~nm}$ bandwidth (full-width at half maximum) [8-17].

During in vivo measurements, no sensation of the light or sound impinging on the skin was felt. The sinusoidal driving wave was amplified using a power supply and then a speaker was placed near the tissue to vibrate the skin. The amplified sound could be heard during the examination but the sound was never above 60 decibels.

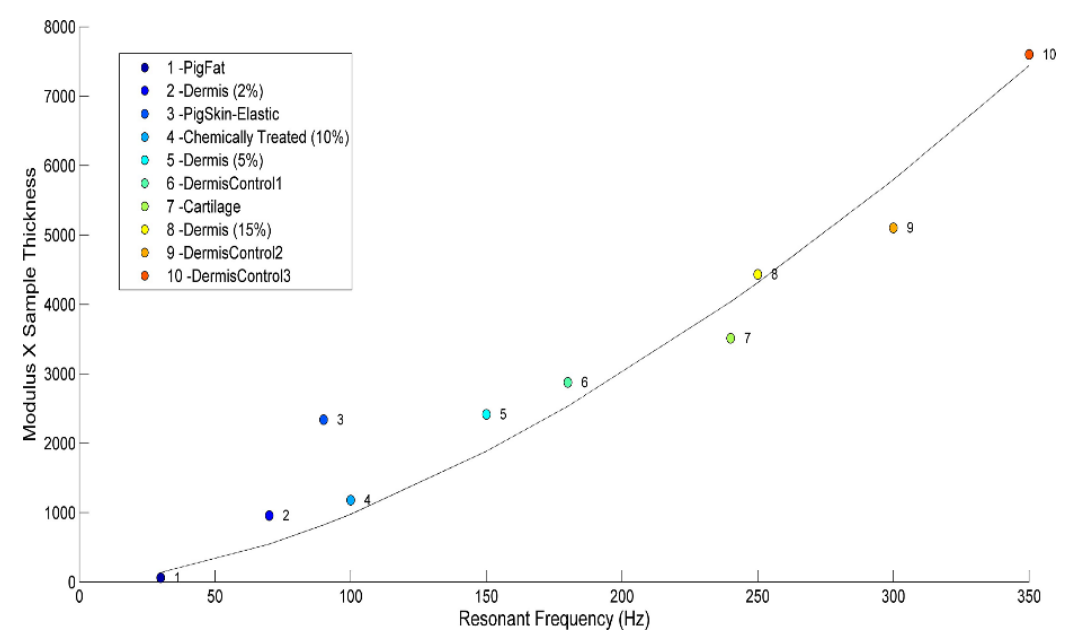

Figure 3: Calibration curve of tissue thickness times modulus versus resonant frequency. This curve was obtained from in vitro calibration studies using a variety of extracellular matrices [8-17].

The resonant frequency of each sample was initially estimated by measuring the transverse displacement resulting from sinusoidal driving frequencies ranging from $30 \mathrm{~Hz}$ to $300 \mathrm{~Hz}$, in steps of $50 \mathrm{~Hz}$. Once the region where the maximum 
displacement was identified, smaller steps of $10 \mathrm{~Hz}$ were used to more accurately identify the peak frequency (the resonant frequency), $f_{n}$. The moduli of skin and skin lesions were calculated from measurements of the resonant frequency and tissue thickness made using VOCT and images of the tissues. Moduli were then obtained from a calibration curve that relates resonant frequency and thickness to modulus values (Figure 3).

\section{Results}

\section{Imaging Normal Skin and Skin Lesions}

In normal skin, the epidermis is quite thin and the cellular layers are visible after color coding the image based on pixel intensities as shown in Figure 4. The stratum corneum (C), granulosum (G), lucidum (L) and Basale (B), and the papillae in the dermis $(\mathrm{P})$ are labeled in the enhanced OCT image as reported previously for normal skin [17,20]. Figure 2 shows the resonant frequencies of normal skin and scar tissues. The calculated modulus of thermal scar tissue is much higher (Table 1) than that of normal skin in vivo. The epidermis of skin and scar are too thin to measure the resonant frequencies of these layers [17,20]. However, if the epidermis proliferates the resonant frequency of the epidermis can be measured as recently demonstrated for skin lesions $[17,20]$. The enhanced OCT image of a congenital nevus is shown in Figure 5. Note the image thickness of the congenital nevus epidermis and dermis is much thinner than that of normal skin suggesting that light penetration and reflection from the nevus is limited compared to that of normal skin. The resonant frequency and modulus values obtained by VOCT for normal skin and the congenital nevus are shown in Table 1. Note because of the light absorption by the congenital nevus the modulus cannot be calculated correctly until a correction can be made for the impaired light penetration observed.

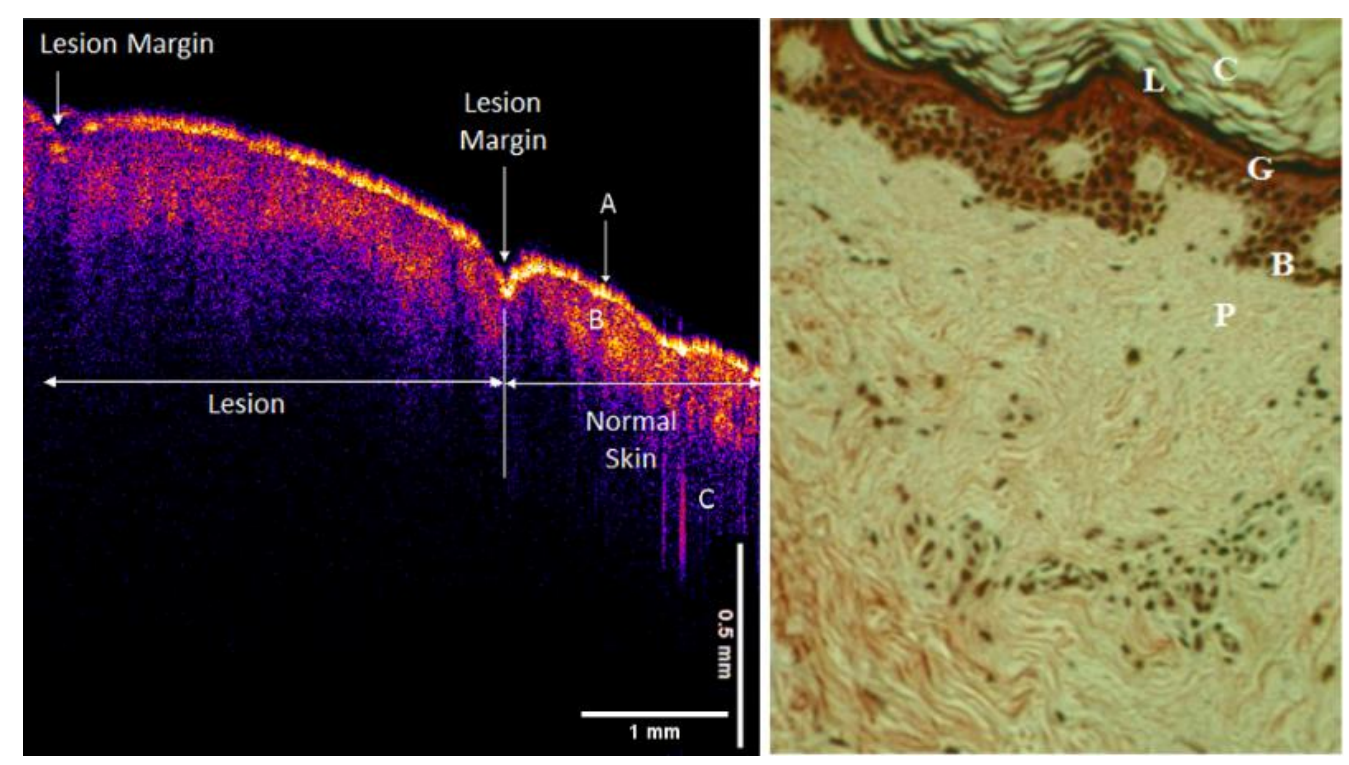

Figure 4: Enhanced OCT image of normal skin and adjacent scar tissue (left) and a histological section of human skin (right). Note the thinness of the stratum corneum and upper layers of skin and scar tissue (lesion) as compared to skin lesions discussed in Figure 6 - Figure 8 [17,20]. The layers of normal skin in the OCT mage include the stratum corneum (A), stratum germinosum (B), and papillary dermis (C). In the histological section (right) the layers include the stratum corneum $(C)$, stratum lucidum $(L)$, stratum germinosum $(G)$, stratum basale $(B)$ and the papillary dermis $(P)$. 


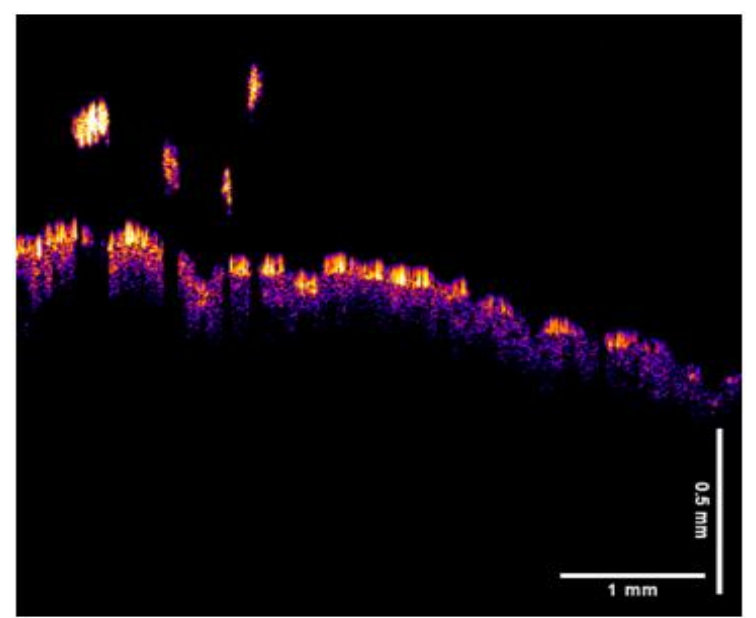

Figure 5: Enhanced OCT image of a congenital nevus. The small image thickness illustrates the lack of penetration of the infrared light due to absorption by melanin of the nevus.

\begin{tabular}{|l|r|r|r|}
\hline \multicolumn{1}{|c|}{ Tissue } & Resonant Frequencies (Hz) & Modulus \#1 (MPa) & Modulus \#2 (MPa) \\
\hline Skin & $90-120^{*}$ & None & 2.0 to 3.0 \\
\hline Scar & $220-230$ & None & 7.0 \\
\hline Melanocytic Nevus & $40,140-180$ & 0.398 & 3.10 \\
\hline Congenital Nevus & 120 & None & $*$ \\
\hline Basal Cell Carcinoma & 60,160 & 0.618 & 2.66 \\
\hline Actinic Keratosis & $70,140-180$ & 0.890 & 3.05 \\
\hline
\end{tabular}

Table 1: The resonant frequency and moduli of skin and different skin lesions collected from [8-17,20] and determined in this study. Note: *exact value will depend on skin thickness correction due to light absorption by melanin.

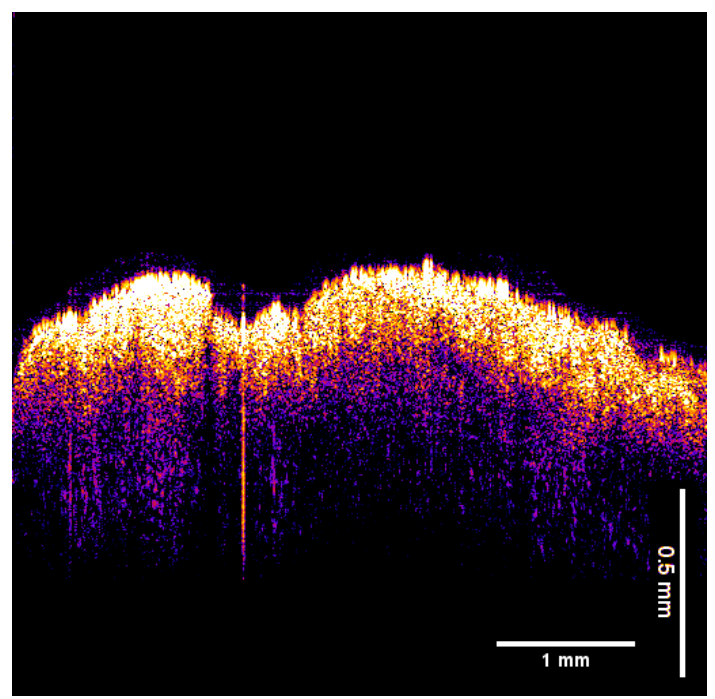

Figure 6: Enhanced OCT image of a melanocytic nevus. Note the increased depth of penetration of the light when compared to Figure 5. This difference is associated with the absorption of light by large amount of melanin contained in the congenital nevus. 
Figure 6 - Figure 8 shows the enhanced OCT images for a melanocytic nevus, an actinic keratosis, and a basal cell carcinoma. Note the rete pegs characteristic of normal skin is not seen in the actinic keratosis, a precursor of squamous cell cancer [17,20] and in the basal cell carcinoma $[17,20]$. Note the increased pixel intensity (bright yellow color) in the basal cell carcinoma, actinic keratosis and melanocytic nevus compared to normal skin and congenital nevus.

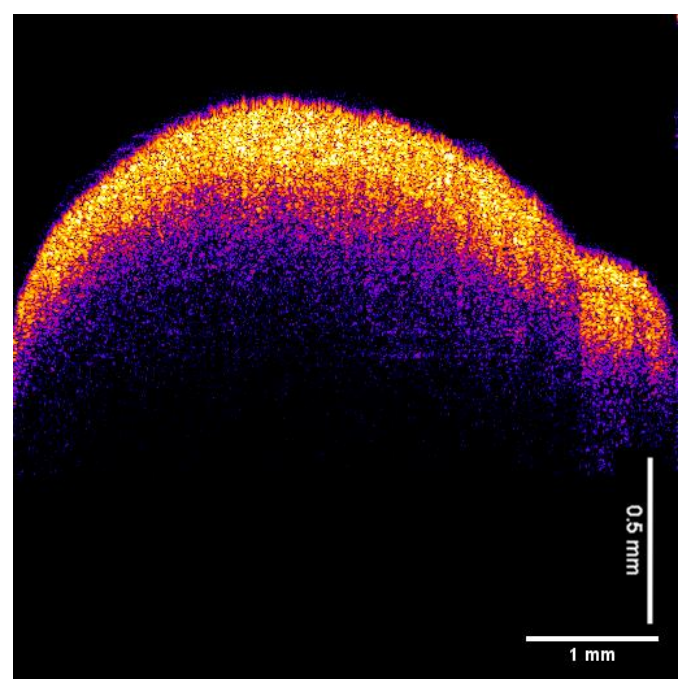

Figure 7: Enhanced OCT image of a basal cell carcinoma (BCC). Note the bright yellow upper layers of the epidermis when compared to Figure 4. The increased brightness compared to Figure 4 suggests that decreased light absorption is due to decreased cytochrome $\mathrm{c}$ oxidase activity that occurs in cancerous tissues.

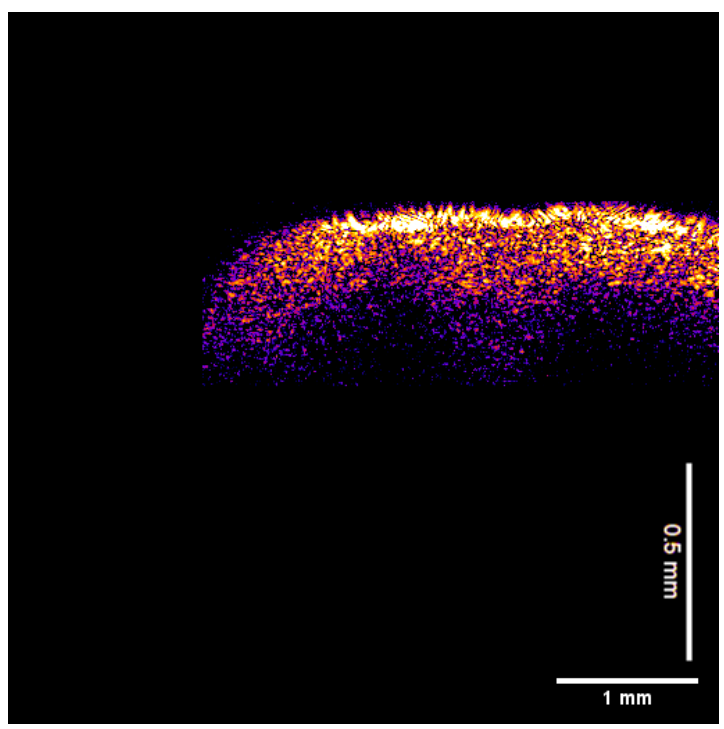

Figure 8: Enhanced OCT image of an actinic keratosis lesion. Note the intermediate brightness of the upper epidermal layers between that of normal skin (Figure 4) and the basal cell carcinoma (BCC) (Figure 7) suggesting that some decrease in cytochrome c activity may occur in this lesion compared to normal skin Figure 7.

\section{Discussion}

We have developed a method termed vibrational optical coherence tomography (VOCT) to do virtual biopsies on skin and skin lesions. The VOCT image can be color enhanced to see all the layers of skin and skin lesions as reported previously [8$17,20]$. In addition, it is possible to measure the stiffness of the cellular and collagenous components of skin by measuring the 
http://www.tridhascholars.org | October-2019

resonant frequency of these components after a transverse sinusoidal sound wave is applied to the surface of the skin [8$17,20]$.

In this study, we ask the question whether we can obtain additional information concerning the layers that make up skin and skin lesions by determining how deep the infrared light (wavelength of $810 \mathrm{~nm}$ ) can penetrate. In theory, we can also measure the relative pixel intensities that result from the reflection of the light. To answer this question, we must consider what factors affect the reflection and absorption of infrared light that occur during our OCT measurements.

Incident light hitting the sample is absorbed, reflected or scattered by surface. While the "optical window" for skin has been reported to be between 600 and $1350 \mathrm{~nm}$ [20, 21], both melanin and cytochrome c oxidase have been reported to absorb light at about $810 \mathrm{~nm}$ [4, 21, 22]. For light colored human skin, the reflectance of light is as high as $90 \%$ at a wavelength of 760 $\mathrm{nm}$; for dark colored skin it decreases to 0.4 depending on the melanin content [20].

Absorption of light by melanin in melanocytes and by cytochrome c oxidase found in mitochondria located in adherens junctions reduces the amount of light reflected back to the OCT device and decreases the depth to which the light can penetrate. Increased production of cytochrome c oxidase is observed during normal apoptosis of cells [4, 22] and is reported to accelerate skin healing [4]. Apoptosis resistance and cancer are associated with decreased levels of cytochrome c oxidase that are induced by transformed cells [22]. The stability of mitochondrial ion channels and the lack of release of cytochrome c into cellular adherence junctions is also associated with chemo-resistance of cancer cells [23].

These study results indicate that light penetration through the skin is decreased in the congenital nevus due to absorption by melanin (Figure 5) while in comparison the light intensity in the melanocytic nevus is increased (increased yellow in Figure 6) and appears to indicate that the amount of melanin in the cells of the lesion is not enough to offset the increased reflected light intensity due to cytochrome c oxidase activity reduction. Furthermore, in the images of the melanocytic nevus (Figure 6), basal cell carcinoma (Figure 7) and the actinic keratosis (AK) (Figure 8) the reflected light (bright yellow) in the superficial part of the epidermis appears to be greater than that observed with normal skin or scar tissue. Especially in the case of the basal cell carcinoma (BCC), the bright yellow color appears to indicate that cytochrome c oxidase absorption of light is impaired. The increased pixel intensity seen in BCC suggests that cytochrome c oxidase activity is reduced and this reduction may be a measure of the malignancy of a lesion if it can be quantitatively assessed.

In contrast to the melanocytic nevus, the congenital nevus did not demonstrate a cellular peak as demonstrated by the data in Table 1. The melanocytic nevus, BCC and AK lesions all had both cellular and collagen peaks as reported previously [17, 20, 24].

\section{Conclusion}

Both the presence of melanin and cytochrome c oxidase decreases the amount of reflected light at a wavelength of $810 \mathrm{~nm}$. In normal skin, the epidermis is qualitatively not as bright as it is in skin lesions including the basal cell carcinoma and actinic keratosis studied. This is due to a lower cytochrome c oxidase activity in these lesions. Cytochrome c oxidase has been shown to be associated with maintenance of normal adherence junction mitochondrial activity required for apoptosis. Apoptosis resistance is considered a hallmark of cancer cells. Therefore, a quantitative comparison of the pixel intensity of normal epithelium and those observed in skin lesions may provide an indicator of cellular function. However, interpretation of the 
http://www.tridhascholars.org | October-2019

enhanced OCT image of "normal skin" and skin lesions depends on understanding how the presence of melanin and cytochrome c oxidase quantitatively affects the pixel intensities of the epidermis and dermis. Additional work is ongoing to understand how to correct pixel intensities of the epidermis and dermis for variation in the amount of melanin present in skin.

\section{References}

1. Bakos RM, Blumetti TP, Roldán-Marín R, et al. (2018) Noninvasive imaging tools in the diagnosis and treatment of skin cancers. American Journal of Clinical Dermatology 19(1): 3-14.

2. Wang J, Xu Y, Boppart SA (2017) Review of optical coherence tomography in oncology. Journal of Biomedical Optics 22(12): 121711.

3. Polańska A, Dańczak-Pazdrowska A, Jałowska M, et al. (2017) Current applications of high-frequency ultrasonography in dermatology. Advances in Dermatology and Allergology 34(6): 535-542.

4. Gupta A, Dai T, Hamblin MR (2014) Effect of red and near-infrared wavelengths on low-level laser (light) therapyinduced healing of partial-thickness dermal abrasion in mice. Lasers in Medical Science 29(1): 257-265.

5. Pilat P, Borzęcki A, Jazienicki M, et al. (2018) Skin melanoma imaging using ultrasonography: a literature review. Advances in Dermatology and Allergology 35(3): 238-242.

6. Que SKT (2016) Research techniques made simple: noninvasive imaging technologies for the delineation of basal cell carcinomas. Journal of Investigative Dermatology 136(4): e33-e38.

7. Russo T, Piccolo V, Lallas A, et al. (2017) Dermoscopy of malignant skin tumours: what's new?. Dermatology 233(1): 64-73.

8. Shah RG, Pierce MC, Silver FH (2017) Morphomechanics of dermis-A method for non-destructive testing of collagenous tissues. Skin Research and Technology 23(3): 399-406.

9. Shah RG, Silver FH (2017) Viscoelastic behavior of tissues and implant materials: estimation of the elastic modulus and viscous contribution using optical coherence tomography and vibrational analysis. Journal of Biomedical Technology and Research 3: 105-109.

10. Shah R, Pierce MC, Silver FH (2017) A method for nondestructive mechanical testing of tissues and implants. Journal of Biomedical Materials Research Part A 105(1): 15-22.

11. Silver FH, Silver LL (2017) Non-invasive viscoelastic behavior of human skin and decellularized dermis using vibrational OCT. Dermatology Clinics and Research 3(3): 174-179.

12. Shah RG, DeVore D, Silver FH (2018) Biomechanical analysis of decellularized dermis and skin: Initial in vivo observations using optical cohesion tomography and vibrational analysis. Journal of Biomedical Materials Research Part A, 106(5): 1421-1427.

13. Silver FH, DeVore D, Shah R (2017) Biochemical, biophysical and mechanical characterization of decellularized dermal implants. Materials Sciences and Applications 8(12): 873-888.

14. Silver FH, Shah RG (2019) Mechanical spectroscopy and imaging of skin components in vivo: Assignment of the observed moduli. Skin Research and Technology 25(1): 47-53.

15. Silver FH, Silver LL (2018) Use of Vibrational optical coherence tomography in dermatology. Archives of Dermatology and Skin Care 1(2): 03-08.

16. Silver FH, Shah RG, Benedetto D, et al. (2019) Virtual biopsy and physical characterization of tissues, biofilms, implants and viscoelastic liquids using vibrational optical coherence tomography. World Journal of Mechanics 9: 1-16. 
http://www.tridhascholars.org | October-2019

17. Silver FH, Shah RG, Richard M, et al. (2019) Comparative "virtual biopsies" of normal skin and skin lesions using vibrational optical coherence tomography. Skin Research and Technology.

18. Carrera C, Marghoob AA (2016) Discriminating nevi from melanomas: clues and pitfalls. Dermatologic Clinics 34(4): 395-409.

19. Emon B, Bauer J, Jain Y, et al. (2018) Biophysics of tumor microenvironment and cancer metastasis-a mini review. Computational and Structural Biotechnology Journal 16: 279-287.

20. Silver FH, Shah RG, Richard M, et al. (2019) Comparison of the virtual biopsies of a nodular basal cell carcinoma and an actinic keratosis: morphological, cellular and collagen analyses. Advances in Tissue Engineering and Regenerative Medicine 5(2): 61-66.

21. Dawson JB, Barker DJ, Ellis DJ, et al. (1980) A theoretical and experimental study of light absorption and scattering by in vivo skin. Physics in Medicine \& Biology 25(4): 695-709.

22. Sabino CP, Deana M, Yoshimura TM, et al. (1980) The optical properties of mouse skin in the visible and near infrared spectral regions. Journal of Photochemistry and Photobiology B: Biology 160: 72-78.

23. Hanahan D, Weinberg RA (2011) Hallmarks of cancer: the next generation. Cell 144(5): 646-674.

24. Peruzzo R, Szabo I (2019) Contribution of mitochondrial ion channels to chemo-resistance in cancer cells. Cancers 11(6): 761. 